\title{
Detection of Genetic Variations in Coagulopathy-Related Genes Using Ramified Rolling Circle Amplification
}

\author{
James H. Smith, ${ }^{1}$ Miao Cui, ${ }^{2}$ David Y. Zhang, ${ }^{2}$ Thomas P. Beals, ${ }^{1}$ and Fei Ye ${ }^{2}$ \\ ${ }^{1}$ Research and Development Department, Thorne Diagnostics Inc. 100 Cummings Center, Beverly, MA 01915, USA \\ ${ }^{2}$ Department of Pathology, Icahn School of Medicine at Mount Sinai, 1428 Madison Avenue, New York City, NY 10029, USA \\ Correspondence should be addressed to James H. Smith; jhsmith@thornediagnostics.com
}

Received 12 August 2013; Accepted 17 January 2014; Published 2 March 2014

Academic Editor: Michael Kalafatis

Copyright (c) 2014 James H. Smith et al. This is an open access article distributed under the Creative Commons Attribution License, which permits unrestricted use, distribution, and reproduction in any medium, provided the original work is properly cited.

\begin{abstract}
We evaluated single nucleotide polymorphism (SNP) detection via a target-capture, C-probe ligation, and RAM assay in a singleblind comparison to clinical samples that had been tested with FDA-cleared tests for up to 4 different vascular disease-related SNPs. In the RAM assay circulizable linear probes (C- or padlock probes) were annealed directly to genomic DNA, processed on a largely automated platform, and ligated C-probes were amplified by real-time RAM. After allele determinations were made with the experimental system, the sample genotypes were unblinded and the experimentally determined genotypes were found to be completely consistent with the FDA-cleared test results. The methods and results presented here show that a combination of Cprobes, automated sample processing, and isothermal RAM provides a robust, and specific, nucleic acid detection platform that is compatible with automated DNA sample preparation and the throughput requirements of the clinical laboratory.
\end{abstract}

\section{Introduction}

The ability to distinguish single nucleotide differences between nucleic acid targets, for example, single nucleotide polymorphisms (SNPs), is a stringent challenge for a nucleic acid target detection assay. An assay platform with high specificity in SNP target detection, suggests utility for other applications where targets exhibit larger differences, such as deletions, insertions or substitutions of more than one nucleotide. In an earlier study [1], using commercially obtained control genomic DNAs, we demonstrated that a substantially automated RAM assay specifically detects all three genotypes of a SNP in the FV gene (FV 1691 G-A) [2].

To further evaluate this RAM assay platform we tested additional SNP targets in clinical samples. We chose a clinically relevant panel of 4 SNP targets (FV 1691 G-A [2], PT 20210 G-A [3], MTHFR 677 C-T [4] and MTHFR 1298 A$\mathrm{C}$ [5]) that are associated with coagulopathic disease and that are routinely tested for in our laboratory using commercial FDA-cleared kits.

We tested the aforementioned SNP targets to further assess the performance of the RAM assay on additional targets, with dissimilar nucleotide substitutions, using previously characterized patient samples. Genotype correlation with vascular disease was an early application of genetic diagnosis $[6,7]$ and hundreds of vascular disease candidate genes have now been identified [8].

The Factor V (FV) gene mutation, FV 1691 G-A, results in an altered gene product that is more resistant to cleavage and is associated with venous disease [2]. In the clotting cascade, activated FV binds to activated Factor $\mathrm{X}$ to convert Factor II (prothrombin, (PT)) to an active protease that cleaves fibrinogen to fibrin (reviewed in [9]). The PT 20210 G-A mutation in the PT-encoding gene that results in elevated plasma prothrombin levels [3] is also associated with vascular disease [7].

Mutations in the above-mentioned genes encoding coagulation-related gene products are largely confined to coagulation-related phenotypes. By contrast, isoforms of the methylenetetrahydrofolate reductase (MTHFR) enzyme have been implicated in diverse phenotypes. The MTHFR 677 C$\mathrm{T}$ mutation [4] that results in a thermolabile enzyme and the MTHFR 1298 A-C mutation [5] have been associated with phenotypes ranging from coronary artery disease [6] to miscarriage [10] to diabetes and obesity $[11,12]$. 
DNA amplification from single-stranded DNA (ssDNA) molecules (referred to here as C-probes) that have been circularized on targets has been used in a variety of assay formats. C-probes are linear molecules with target-specific termini separated by an internal spacer sequence or core and have been described by us $[1,13]$ and others [14-17]. Following target-dependent ligation the resulting ssDNA circles can be amplified via ramified DNA amplification using a pair of primers [15, 17], a single primer [18], or by polymerase chain reaction (PCR; [15]).

Here we evaluate the C-probe ligation and RAM amplification assay as previously described [1] for highly specific nucleic acid detection by testing clinical samples for up to 4 SNP targets. Employing capture-probe technology on an automated platform eliminated RAM reaction noise when ssDNA circles were detected by SYBR-Green fluorescence in real-time amplification reactions. In a single-blind comparison, we tested patient genomic DNA samples, from whole blood, for the aforementioned coagulopathy-related polymorphisms. After RAM testing was complete, sample genotypes were scored as wild type (Wt) or mutant $(\mathrm{Mt})$ homozygotes, or as heterozygotes. Sample genotypes were then unblinded and compared to RAM results.

\section{Materials and Methods}

2.1. Methodology Overview. The workflow for the experimental assay was designed to be compatible with the automated whole-blood sample processing protocols employed prior to analysis with commercial assays. Figure 1 shows an overview of the RAM-assay-based process described here; a more detailed description was provided earlier [1]. DNA samples were fragmented and then incubated with SNPspecific C-probes and biotin-tagged capture probes under DNA hybridization conditions as described earlier [1]. After hybridization, binding of the capture probe/target/C-probe ternary complex to streptavidin-coupled magnetic beads, bead-washing, C-probe ligation, and sample suspension in RAM-assay-ready form followed as automated steps. Realtime signals were recorded for each RAM reaction via SYBRGreen fluorescence [13] monitoring.

2.2. Reagents. Hybridization, wash, and elution buffers and ligation, amplification, and bead mixtures were described earlier [1]. The beads were SeraMag Streptavidin Particles (part No. 3015105010150, Thermo Scientific, Indianapolis, IN) with a nominal biotin-binding capacity of 4559 pmol biotin/mg.

\subsection{Nucleic Acids Detection Reagents Design and Quality} Control. All C-probes, capture probes and RAM primers were synthesized by Gene Link Inc. (Hawthorne, NY), and both $\mathrm{C}$-probes and capture probes were gel-purified by the vendor. All sequences are shown in Table 1. Capture probes were modified with a $5^{\prime}$ biotin moiety (Table 1 "Bio-") for streptavidin capture and with a $3^{\prime}$ spacer C3 moiety (Table 1 , “-Spacer C3") to block 3 ' extension by the DNA polymerase. Figure 2(a) depicts conceptually the alignment of the targetspecific termini of a pair of C-probes that detect SNPs

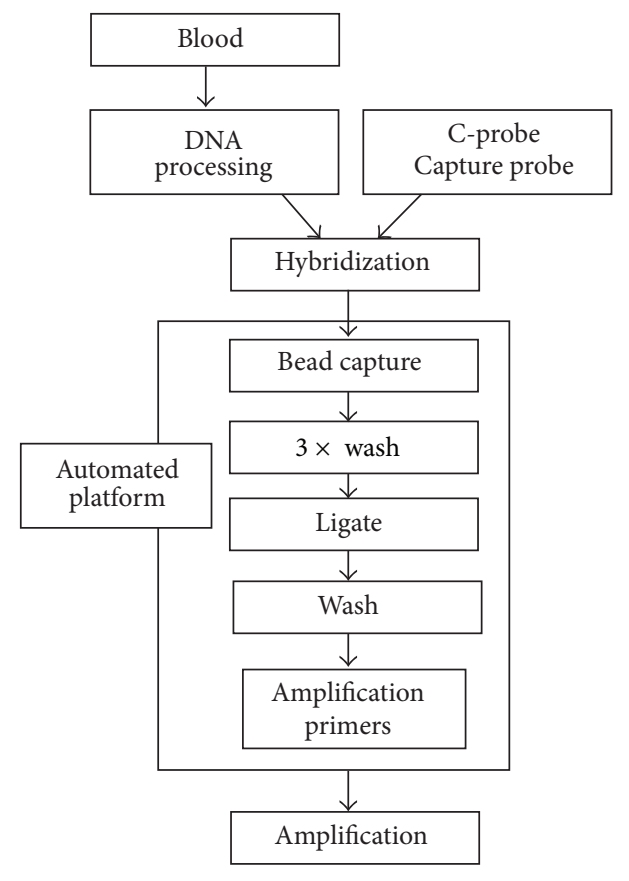

Figure 1: Process diagram for RAM assays. The figure shows conceptually the groups of operations performed as described in the text. "Hybridization" refers to DNA hybridization. "Automated platform" refers to the Kingfisher 96 instrument described in the text.

on the Wt and Mt DNA target strands. 2 strand-specific biotin-labeled capture probes (not shown ([1], Figure 1)) bind to each strand $3^{\prime}$ proximal (downstream) to the Cprobe binding domain. The biotin moiety on the capture probe mediates binding of the ternary complex (target/Cprobe/capture probe) to streptavidin-coated beads.

C-probes with allele-specific termini were designed as described earlier [1]. As described below, there are several options for the design of probe-target recognition and the two employed in this study are illustrated in Figure 2. Cprobe, DNA-target combination designations indicate the target strand (plus or minus strand, or top or bottom strand, etc.) to which the C-probe binds, and a target-allele indicator (here, Wt or $\mathrm{Mt}$ ). We indicate a combination by writing the target form, followed by the letter " $\mathrm{C}$ " to indicate a Cprobe, followed by a target-strand indicator. For example, $\mathrm{WtC}+$ indicates a $\mathrm{C}$-probe that binds the target plus-strand to detect a wild-type genotype. Multiple pairings are commaseparated; thus, the combination of $\mathrm{WtC}+, \mathrm{MtC}-$ adds to the above a mutant-detecting $\mathrm{C}$-probe that is complementary to the "minus" target strand (Figure 2(a)) and a second combination, $\mathrm{MtC}+, \mathrm{WtC}-$, is illustrated in Figures 2(b) and 2(c). For another example, an alternate version of the assays described here could utilize a single capture-probe and two C-probes that are specific for alternate forms of one target strand: $\mathrm{WtC}+, \mathrm{MtC}+$ (configuration not shown). The potential advantage of these options is described in the Discussion Section. 


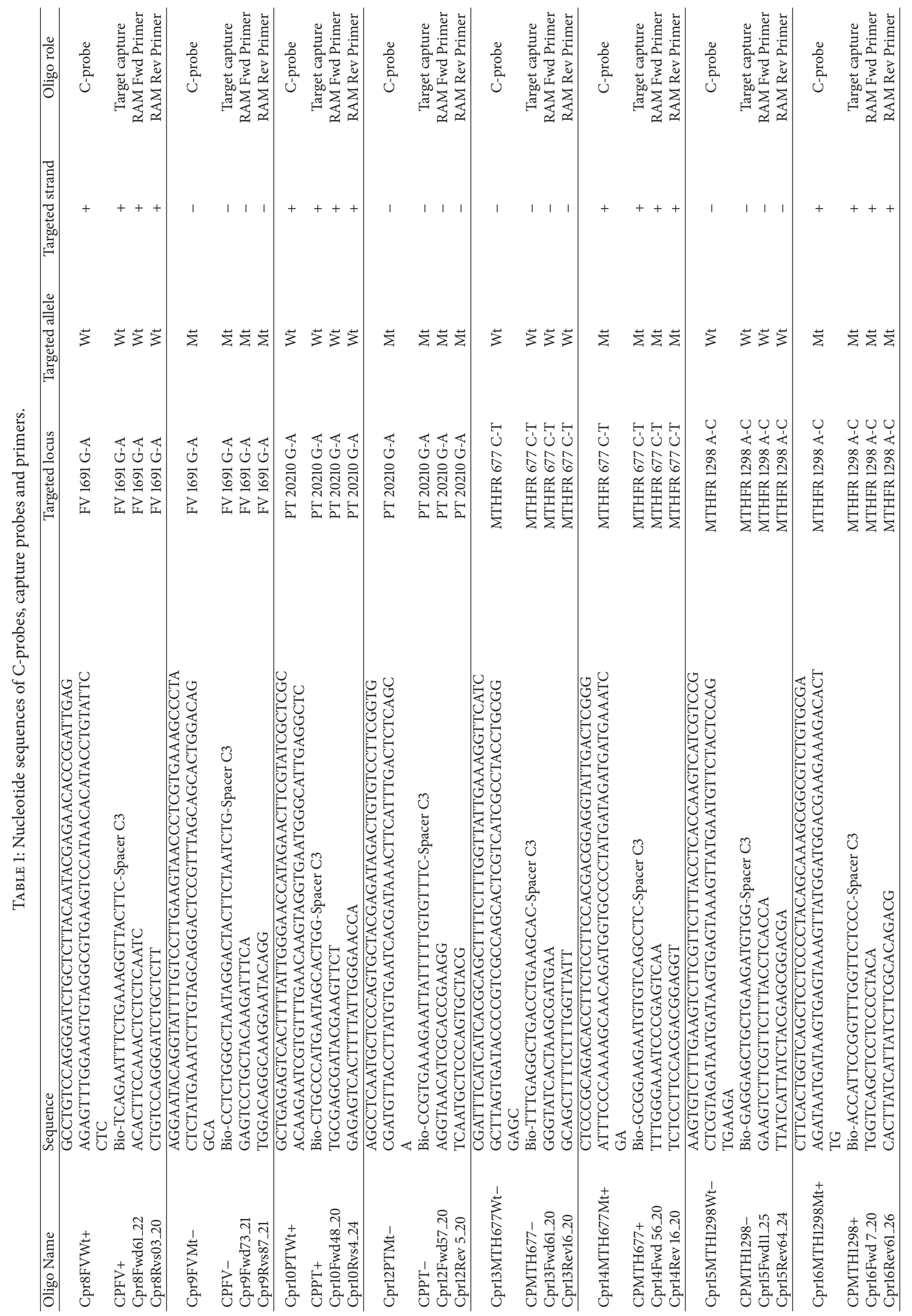


FV 1691 G-A and PT 20210 G-A (G>A)

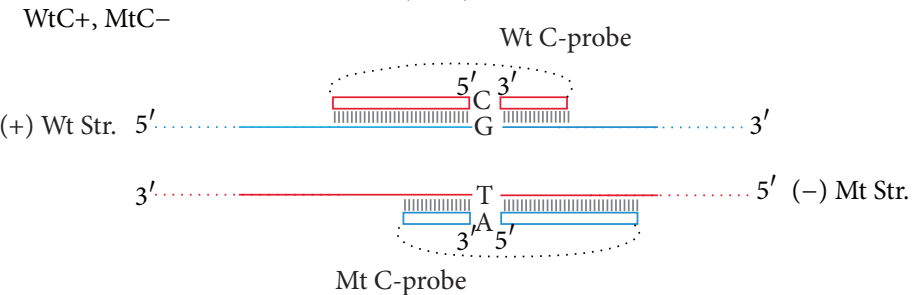

(a)

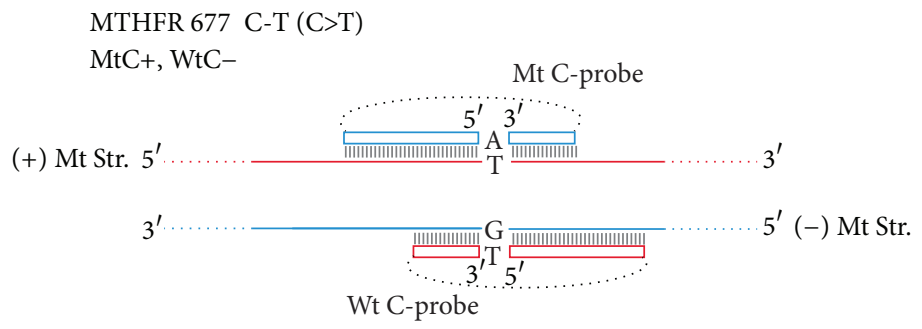

(b)

MTHFR 1298 A-C $($ A $>C)$

$\mathrm{MtC}+, \mathrm{WtC}-$

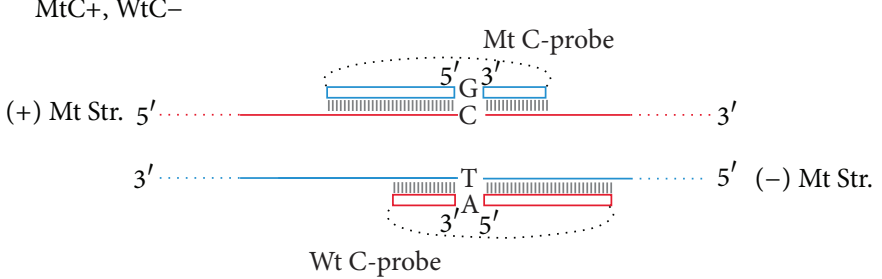

(c)

FIGURE 2: The alignment of C-probe target specific termini that detect SNP targets via the RAM reaction. The figure shows Wt target strands ("Wt Str.") and Mt C-probe target-specific terminal sequence regions in blue and the Mt target strands ("Mt Str.") and Wt C-probe terminal target specific regions in red. Wt and Mt C-probe termini are shown as if bound to their respective targets, and joined by a target-nonhomologous linker represented by a dotted line. The SNP base is shown in bold-face in the target strands and on the $3^{\prime}$ terminal base of each C-probe. Strand specific capture probes (not show) bind proximally downstream ( $3^{\prime}$ ) of the C-probe binding domain. (a) shows the FV and $\mathrm{PT}$ target/probe arrangement, $\mathrm{WtC}+, \mathrm{MtC}-$, and (b and c) the MtC+, WtC- format used for the detection of both MTHFR SNPs, MTHFR 677 C-T and MTHFR 1298 A-C respectively.

The C-probe design-phase included compatible primer specification; initial primers were selected using Primer3 [19]. Selected C-probes and primers were synthesized and primer pairs were tested in real-time RAM assays with preformed circularized C-probes as templates (data not shown). Cprobe concentrations in hybridization reactions (Table 2) reflect adjustments for specific C-probe performance and for synthesis-batch-specific C-probe characteristics as described earlier [1]. Primer pair concentrations in RAM reactions (Table 2) reflect synthesis batch-specific optimization and reaction rate characteristics, as described previously [1].

Biotin-linked capture probes provide an additional measure of specificity by annealing to a defined sequence flanking the SNP locus of interest. The target-specific capture probe sequences (Table 1) are designed to bind to target strands nonpreferentially with respect to the SNP locus genotype.

2.4. Clinical Samples, DNA Preparation, and Control DNA. Blood samples were obtained from patients presenting for thrombosis or related conditions at the Molecular Pathology
Laboratory, Icahn School of Medicine at Mount Sinai. The governing Institutional Review Board determined that this work was not subject to regulation as determined by Department of Health and Human Services and Food and Drug Administration policies. Patients' DNA was isolated from $50 \mathrm{uL}$ (PT and FV) or $200 \mathrm{uL}$ (MTHFR) of blood using a Magnapure LC Instrument (Roche Applied Science, Indianapolis, IN) and QIAamp DNA blood mini kit (QIAGEN, Valencia, CA), respectively. DNA was eluted in 100 uL elution buffer (Magnapure) or $200 \mathrm{uL}$ elution buffer (QIAGEN). Typical DNA concentrations were from 10 to $20 \mathrm{ug} / \mathrm{mL}$ [20].

Controls were obtained from the Coriell Institute for Medical Research (Camden, NJ). Coriell catalog ID numbers and the corresponding C-probes used for SNP detection are listed in Table 3. For control assays (Figure 3), $4 \times$ $10^{4}$ genome-equivalents of Coriell DNAs were used per hybridization.

We expect from the central limit theorem that C-probe circle numbers in postligation control samples are normally distributed; since response time is a logarithmic function 
TABLE 2: C-probe and primer concentrations used in hybridizations and RAMs respectively. Primer naming conventions are given in [13].

\begin{tabular}{|c|c|c|c|c|}
\hline Allele & C-Probe & $\begin{array}{l}\text { C-Probe Concentration in } \\
2.5 \mathrm{X} \text { buffer }(\mathrm{nM})\end{array}$ & Primer pair & $\begin{array}{l}\text { Primer concentration } \\
\text { (uM) in elution buffer }\end{array}$ \\
\hline \multirow{2}{*}{ FV 1691 G-A Wt } & \multirow{2}{*}{ Cpr8FVWt+ } & \multirow{2}{*}{0.4} & Cpr8FVFwd61_22 & 2.5 \\
\hline & & & Cpr8Rvs03_20 & 2.2 \\
\hline \multirow{2}{*}{ FV 1691 G-A Mut } & \multirow{2}{*}{ Cpr9FVMt- } & \multirow{2}{*}{0.25} & Cpr9Fwd73_21 & 2.5 \\
\hline & & & Cpr9Rvs87_21 & 1.5 \\
\hline \multirow{2}{*}{ PT 20210 G-A Wt } & \multirow{2}{*}{ Cpr10PTWt+ } & \multirow{2}{*}{0.1} & Cpr10Fwd48_20 & 1.7 \\
\hline & & & Cpr10Rvs04_24 & 1 \\
\hline \multirow{2}{*}{ PT 20210 G-A Mut } & \multirow{2}{*}{ Cpr12PTMt- } & \multirow{2}{*}{0.125} & Cpr12Fwd57_20 & 3 \\
\hline & & & Cpr12Rev05_20 & 1.8 \\
\hline \multirow{2}{*}{ MTHFR 677 C-T Wt } & \multirow{2}{*}{ Cpr13MTH677Wt- } & \multirow{2}{*}{0.25} & Cpr13Fwd61_20 & 3.6 \\
\hline & & & Cpr13Rev16_20 & 2.2 \\
\hline \multirow{2}{*}{ MTHFR 677 C-T Mut } & \multirow{2}{*}{ CPMTH677Mt+ } & \multirow{2}{*}{1.25} & Cpr14Fwd56_20 & 2.5 \\
\hline & & & Cpr14Rev 16_20 & 1.5 \\
\hline \multirow{2}{*}{ MTHFR 1298 A-C Wt } & \multirow{2}{*}{ Cpr15MTH1298Wt- } & \multirow{2}{*}{0.125} & Cpr15Fwd11_25 & 1.2 \\
\hline & & & Cpr15Rev64_24 & 0.7 \\
\hline \multirow{2}{*}{ MTHFR 1298 A-C Mut } & \multirow{2}{*}{ Cpr16MTH1298Mt+ } & \multirow{2}{*}{0.25} & Cpr16Fwd 7_20 & 1.4 \\
\hline & & & Cpr16Rev61_26 & 0.9 \\
\hline
\end{tabular}

All capture probes concentrations in the $2.5 \mathrm{X}$ buffer were $2.5 \mathrm{nM}$.

TABLE 3: List of control DNAs and corresponding C-Probes used for SNP detection.

\begin{tabular}{lccccr}
\hline Coriell catalog & Coriell nucleic acid & C-probe & Locus & Allele & ThDx allele state \\
\hline GM00536 & NA00536 & Cpr8 & FV 1691 G-A & Wt & Homozygous, Wt \\
GM14899 & NA14899 & Cpr9 & FV 1691 G-A & Mut & Homozygous, Mt \\
GM00536 & NA00536 & Cpr10 & PT 20210 G-A & Wt & Homozygous, Wt \\
GM16000 & NA16000 & Cpr12 & PT 20210 G-A & Mut & Homozygous, Mt \\
GM03469 & NA03469 & Cpr13 & MTHFR 677 C-T & Wt & Homozygous, Wt \\
CD00024 & CD00024 & Cpr14 & MTHFR 677 C-T & Mut & Homozygous, Mt \\
GM08369 & NA08369 & Cpr15 & MTHFR 1298 A-C & Wt & Homozygous, Wt \\
GM00536 & NA00536 & Cpr16 & MTHFR 1298 A-C & Mut & Homozygous, Mt \\
GM16028 & NA16028 & Cpr8 & FV 1691 G-A & Wt & Heterozygous \\
GM16028 & NA16028 & Cpr9 & FV 1691 G-A & Wt & Heterozygous \\
GM16028 & NA16028 & Cpr10 & PT 20210 G-A & Mut & Heterozygous \\
GM16028 & NA16028 & Cpr12 & PT 20210 G-A & Wt & Heterozygous \\
GM16028 & NA16028 & Cpr13 & MTHFR 677 C-T & Mut & Heterozygous \\
GM16028 & NA16028 & Cpr14 & MTHFR 677 C-T & Wt & Heterozygous \\
GM16000 & NA16000 & Cpr15 & MTHFR 1298 A-C & MTHFR 1298 A-C & Mut \\
GM16000 & NA16000 & Cpr16 & Heterozygous \\
\hline
\end{tabular}

of circle number [13] we fit control response times to a log-normal distribution. Statistical evaluation was done in the $\mathrm{R}$ statistical computing environment [21]. Control data sets were initially evaluated for log-normality by a ShapiroWilks test; data sets with $P$ values less than 0.05 were tested for outliers by Grubbs' method (http://www.CRAN.Rproject.org/package $=$ outliers [22]). Grubbs test data points with $P$ values less than 0.05 were identified as outliers and removed, and then the data was retested.

The genotypes of the clinical samples had been determined using the following commercial assay reagent kits. FV and PT genotypes were determined using Factor V Leiden
(FVL) (Leiden FV 1691 G-A) and Factor II (Prothrombin PT 20210 G-A) kits (Roche Diagnostics, Indianapolis, IN) respectively. These tests are performed by real-time PCR assays followed by melting curve analysis with fluorescence resonance energy transfer (FRET) probes targeted at the FVL or PT mutation sequences. MTHFR 677 C-T and MTHFR 1298 A-C mutation testing were performed using Hologic's Invader Assay from Third Wave Technologies (Madison, WI).

2.5. Sample Processing for RAM Assays. $66 \mathrm{uL}$ DNA, eluted as described above, was digested in $300 \mathrm{uL}$ of $1 \mathrm{X}$ New England Biolabs (NEB, Ipswich, MA) restriction enzyme buffer 


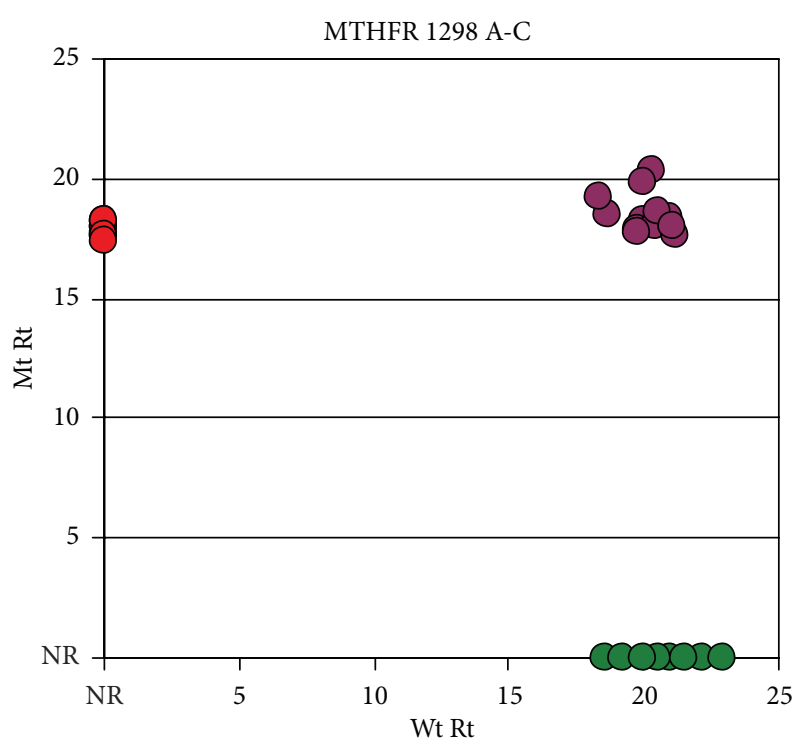

(a)

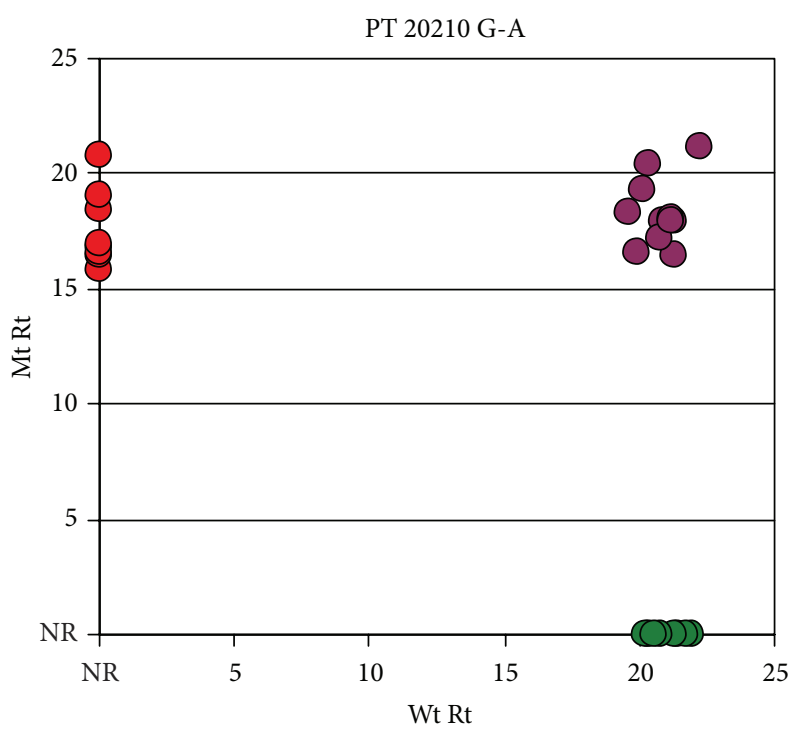

(c)

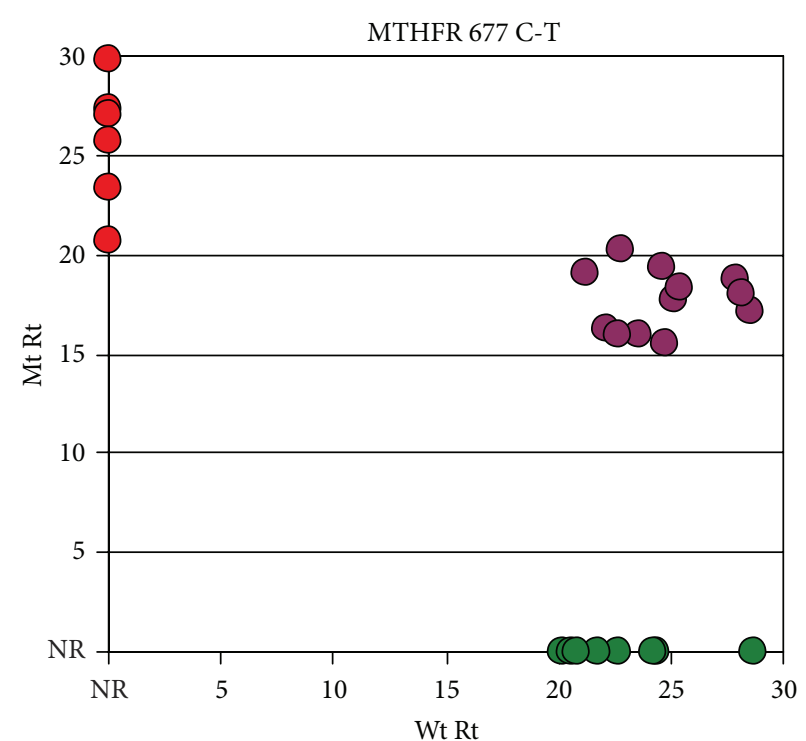

(b)

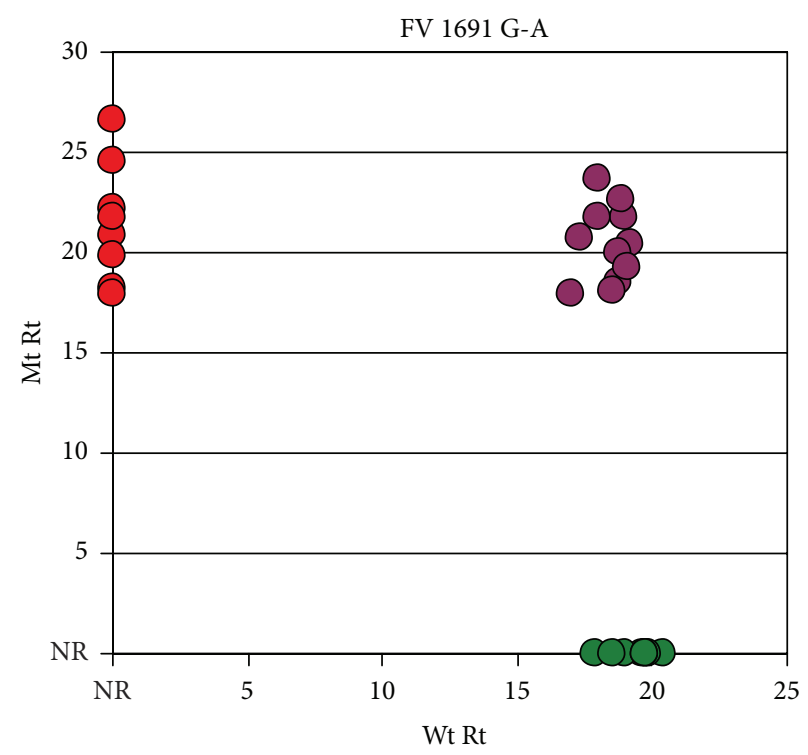

(d)

FIGURE 3: Representative plots of RAM assays performed on control DNA samples. The data points represent RAM signals (Rts) from Cprobes ligated to $\sim 6 \times 10^{3}$ genome equivalents. Green data points represent Wt samples (wild type response times, "Wt Rt"); red data points represent homozygous Mt samples samples (mutant response times, "Mt Rt"); purple data points represent heterozygotes. "NR" on the axis scales represents "No Response". Each homozygous sample response time is plotted on its labeled axis and at the no-response level on the alternate axis; for example, wild type (Wt) data points are plotted at on the "Wt" axis and at "NR" on the "Mt" axis. "Rt", response time. MTHFR 677 C-T "Mt" homozygous samples done in a separate assay have a higher mean response time than the same C-probes applied to the heterozygous samples. Separate measurements for each allele from heterozygous samples are plotted within the panels as example pairings of allele measurements.

4 containing 30 units/mL of BsaI, BspHI, FokI and HaeIII (NEB) at $37^{\circ} \mathrm{C}$ for 1 hour, leaving targets of interest on DNA fragments ranging from 149 to 344 nucleotides. Samples were then denatured at $95^{\circ} \mathrm{C}$ for 10 minutes. $34 \mathrm{uL}$ aliquots of the digest per individual assay were combined, with mixing, with $23 \mathrm{uL}$ of $2.5 \mathrm{x}$ hybridization buffer [1] containing C-probes and capture-probes, at concentrations specified in Table 2 , in the wells of a 96-well microtiter plate. The plate was sealed and held for DNA hybridization at $52^{\circ} \mathrm{C}$ for one hour.
2.6. Magnetic Beads. Preparation of magnetic beads derivatized with streptavidin was performed according to Smith and Beals, 2013 [1]. $50 \mu \mathrm{L}$ of beads, resuspended to $0.025 \%$ solids in 1x hybridization buffer, were added per well of a bead source plate. The final bead concentration at the binding step was $0.022 \%$.

2.7. Elution Plate. Elution plates were prepared by adding $50 \mu \mathrm{L}$ of low salt elution buffer containing C-probe-specific 
primers to each well. The primer concentrations for the elution buffers are shown in Table 2 .

2.8. Automated Sample-Processing. Post-hybridization steps (binding, washing, ligation, re-washing and elution) were carried out on an automated sample-processing platform (Kingfisher 96, Thermo Fisher Scientific Inc., Newington, $\mathrm{NH})$ as described earlier [1]. In the final step on the KingFisher the ligated circles were separated from the beads in low ionic strength elution buffer containing RAM primers; the magnetic beads were subsequently discarded leaving circularized C-probes in the eluate.

2.9. RAM Amplification. $10 \mathrm{uL}$ from the eluate wells was combined with $10 \mathrm{uL}$ RAM reaction mix [1] containing SYBRGreen dye (Molecular Probes, Eugene, OR). In this series of experiments approximately one fifth of the eluate was tested first, allowing duplicate amplifications to be performed on each sample to allow for systems diagnostics. Isothermal $\mathrm{RAM}$ reactions were performed at $63^{\circ} \mathrm{C}$ for 90 minutes in an iCycler (Bio-Rad, Hercules, CA) real-time fluorescence reader. Since the RAM reaction is not a cyclic process, we interpreted the cycle threshold $(\mathrm{Ct})$, as reported by the default settings of the $\mathrm{iCycler} \mathrm{iQ}$ version 3.1 software, as a response time (Rt; $[13,23])$.

2.10. RAM Response-Time Postassay Interpretation. Response time quality control seeks to assess whether response times are due to variance in sample DNA content and to reject rare responses due to $\mathrm{C}$-probe mismatch ligation of a fraction of C-probes [24]. The assay performance of each DNA preparation is inferred and updated from multiple allele assay results from each DNA sample. For SNP, assays subsequent measurements, in the absence of variation in DNA sample concentration, are expected to be within a two-fold range [1] of the first measurement, because the copy number of heterozygous samples should be half the copy number of homozygous samples.

Late response times that are potential mismatch ligation Rt values were evaluated based on inferred template copy number. Control reactions established a log-linear relation (e.g., [25], Figure 4(b)) between input C-probe circle number and response time, by testing preformed C-probe circle dilutions in RAM assays. The full assay-system's response (data not shown) to several levels of known-genotype genomic DNAs (listed in Table 3) established baselines for expected Rt ranges. Control reactions and Rt versus template copy number standards were constructed for each C-probe, primer-pair combination.

Response times for each C-probe annealing and each sample were scored for acceptance in the following sequence. For the assays described here, where all samples were tested for multiple loci, the first data point was accepted if it was within the expected response-time range, based on control sample data. A response-time within the expected range for any sample and either allele at any locus is taken as initial sample validation, indicating adequate initial DNA yield. Samples were scored as heterozygotes if both the Wt and Mt allele assays yielded acceptable signals. Null results for either Wt or Mt assays and an acceptable signal for the alternate allele indicated a homozygous genotype.

\section{Results}

Aliquots of sample DNA prepared as described were carried through the hybridization and automated assay process steps. Figure 3 shows the results of replicate RAM amplifications carried out on control DNAs. The response times for the Wt and Mt control samples are plotted on horizontal and vertical axes, respectively. Assays, in which genomic DNA that is homozygous at a given locus is hybridized with Cprobes that are specific for an alternate allele at that locus, generally do not produce low-level noise in assays, instead usually resulting in no amplification signal. To indicate a lack of signal, the zero level of the plots in Figures 3 and 4 is labeled "NR" indicating no response.

Signals from Wt C-probes detecting Wt homozygous loci are plotted (Figure 3 ) at the no-response level on the "Mt" axis and at their observed response time on the "Wt" axis. Signals from assays in which Mt-specific C-probes detect homozygous Mt loci are plotted correspondingly on the "Mt" axis. In this study, C-probes for Wt and Mt loci were annealed and amplified separately. Signals from genomic DNAs that are heterozygous for the tested locus are plotted in an off-axis cluster.

We performed the RAM assay after C-probe ligation on 44 patient samples for which one or more SNP assay results had been determined using commercial kits. The RAM assays were done in single-blind fashion in the sense that the full assay comprising C-probe ligation, RAM reactions, and interpretation was performed and scored without knowledge of the commercial assay results. Figure 4 shows graphic representations of the distribution of response-time signals for $\mathrm{Wt}$ response-times (Wt Rt) and Mt response-times (Mt Rt) of assay results for patient samples. To assess the consistency of repeated measures, replicate RAM assays were performed on each C-probe hybridization (shown distinguished by color in Figure 4). The number of late responding wells, out of all amplifications, was less than one percent; those readings were flagged as unrepresentative by the quality-control algorithm.

A total of 280 allele determinations were made for comparison to results obtained from commercial assays. Although replicate RAM reactions were run as an internal consistency check, either of the RAM reaction series could have been used for comparison to obtain the same outcome. Figure 5 shows graphically the results obtained after unblinding the commercial assay results. SNP allele determinations made after C-probe ligation and RAM reaction scoring were completely consistent with the commercial assay results over all reported alleles. The summary in Table 4 shows the results by genotype within each individual SNP category from the 44 patient samples tested. Of the samples tested for the MTHFR, more than half of the outcomes were either heterozygous or homozygous Mt. No homozygous mutants were detected for the FV and PT targets. The distribution of genotypes in Table 4 reflects the allele frequencies of the patient samples 


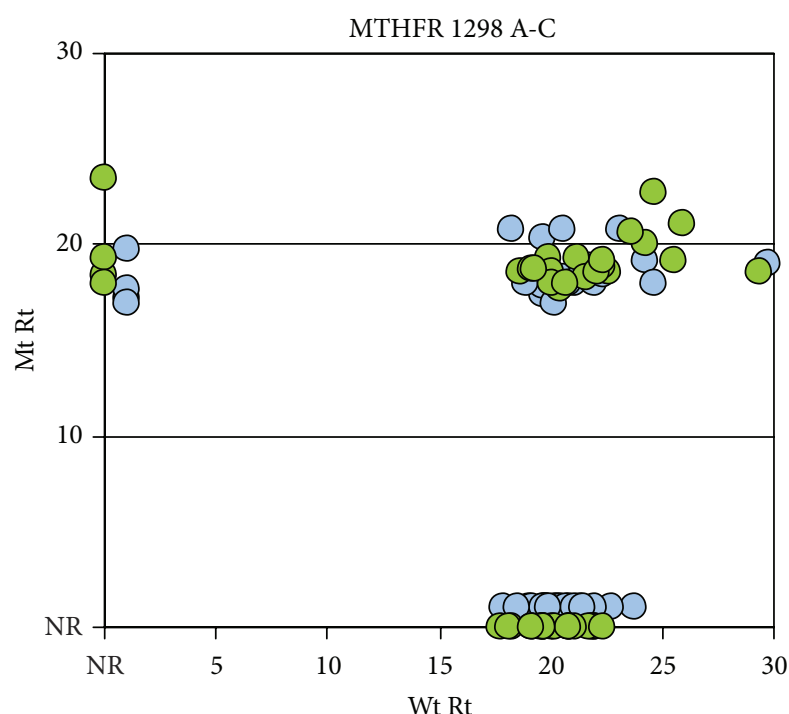

(a)

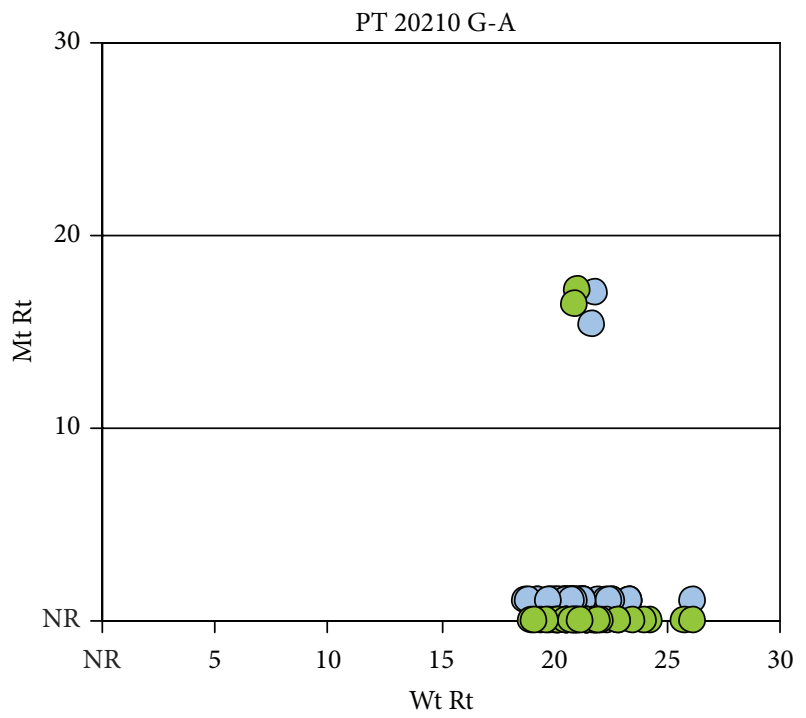

(c)

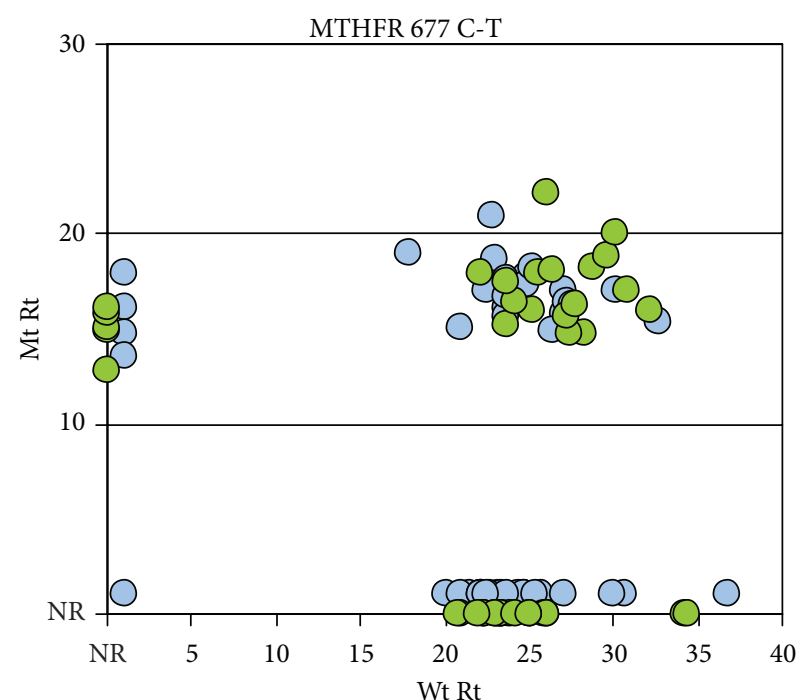

(b)

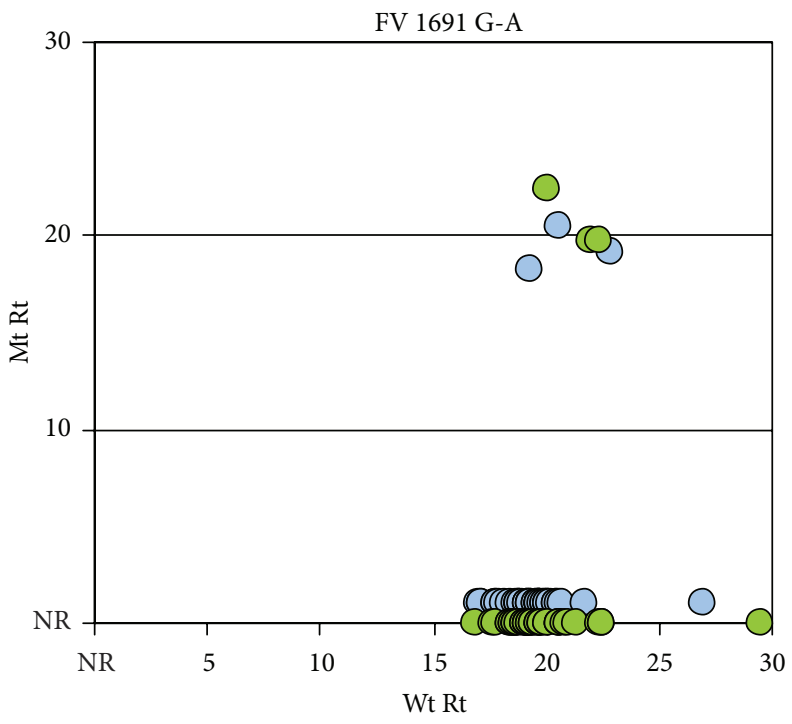

(d)

FIGURE 4: Representative plots of RAM assay results performed on clinical samples. Data points (Rts) from homozygous and heterozygous samples are represented by position as in Figure 3 (homozygous results plotted on the axes and heterozygous results appear off axes), but color distinguishes replicate RAM reactions. No homozygous Mt PT or FV alleles were detected.

received in our laboratory where the MTHFR mutant alleles are more frequent than the FV and PT mutants (data not shown). In the clinical samples tested the Mt probes for FV and PT show good specificity; however, the low number of Mt alleles detected at the FV and PT loci in this study does not allow definitive evaluation of their clinical sensitivity but does demonstrate acceptable sensitivity with the control DNAs (Figure 3).

\section{Discussion}

Earlier we showed that target capture, C-probe hybridization, ligation, and RAM amplification can be configured on an automated platform, and the assay system could
TABLE 4: Summary of assay results by genotype.

\begin{tabular}{lcccc}
\hline SNP target & Wt & Ht & Mt & Totals \\
\hline FV 1691 G-A & $32(94 \%)$ & $2(6 \%)$ & $0(0 \%)$ & 34 \\
PT 200210 A-G & $29(97 \%)$ & $1(3 \%)$ & $0(0 \%)$ & 30 \\
MTHFR 677 C-T & $17(45 \%)$ & $17(45 \%)$ & $4(10 \%)$ & 38 \\
MTHFR 1298 A-C & $17(45 \%)$ & $18(47 \%)$ & $3(8 \%)$ & 38 \\
\hline Totals & & & 140 \\
\hline
\end{tabular}

differentiate a single nucleotide difference in commercially available purified genomic DNAs. To further evaluate the RAM assay, we tested clinical samples of known genotype for 4 individual SNPs in 3 different genes. The 4 discrete SNPs 


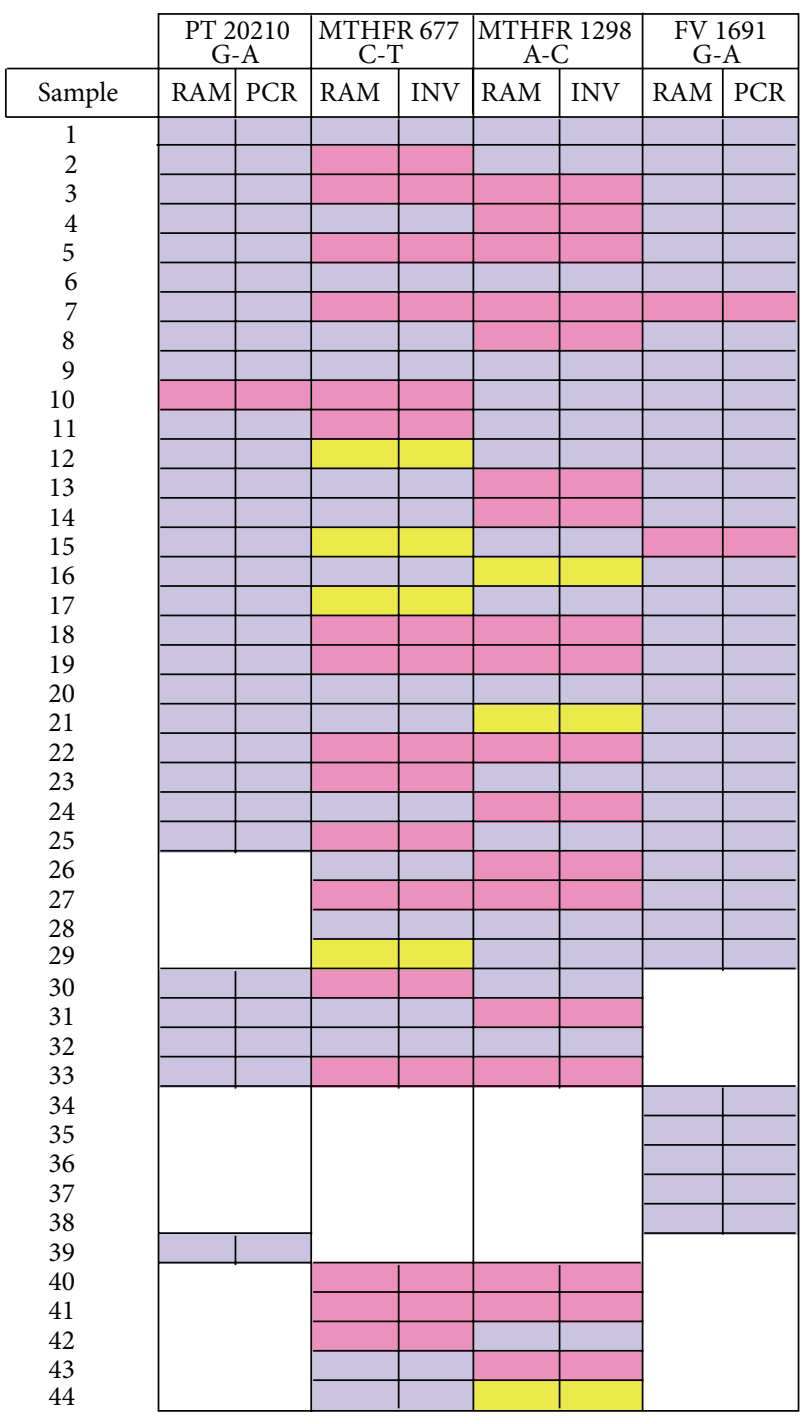

Homozygous Wt

Homozygous Mut

Heterozygous

FIGURE 5: Comparison of genotypes scored after RAM reactions ("RAM") versus genotypes called by commercial assays ("PCR" or Invader, "INV"). Results from each locus are shown in two columns; samples are shown in rows. Colors indicate each sample's genotype at each locus, as assessed by RAM assay (left columns) or FDAcleared assay (right columns). Whitespace indicates that no test was performed on the indicated sample, assay pair.

are represented by 3 different nucleotide base substitutions; two SNPs are guanine to adenine $(\mathrm{G}>\mathrm{A})$ transitions; one is cytosine to thymidine transition $(\mathrm{C}>\mathrm{T})$ and the other is adenine to cytosine transversion $(A>C)$. The format of the assay addresses each allele separately, employing 8 capture and C-probe pairs (Table 1) and utilizing independently both strands of the nucleic acid targets. The rationale for this approach was to achieve the most favorable mismatch, where possible, between the $3^{\prime} \mathrm{C}$-probe nucleotide and the
SNP base. Favorable mismatches minimize the nonspecific ligation of C-probes on nonhomologous targets, which can result in one form of RAM assay noise (discussed below). However, the assay can be simplified in a format where both C-probes address the SNP allele on the same target strand (unpublished data).

The use of a capture probe and bead capture in the assay has several benefits. Clinical samples are generally processed to remove components that would inhibit enzyme activity and thereby impair assay performance [26]. Detergents, especially ionic types, used in sample processing [27, 28], can help liberate and protect targets released from cells. The binding and washing of the captured nucleic acid target complexes substantially reduce detergents, other components, and sample particulates prior to ligation and amplification. Use of a target specific capture probe in combination with a C-probe may also improve the specificity of the assay, particularly if there are closely related target sequences to which the $\mathrm{C}$-probe may bind elsewhere in the genome. In addition, the use of a capture probe and magnetic beads reduces one other form of background noise (discussed below). These advantages were not fully utilized here in a demonstration of consistency with the existing sample processing.

There are several distinguishable nonspecific background noise sources in RAM assays [1]. Careful design and selection of C-probes and their corresponding primers significantly reduce primer interaction noise; in addition, using capture probes and beads in the assay significantly reduces unligated C-probe associated nonspecific RAM reaction product background noise [1].

The ligation of C-probes on nonhomologous SNP targets can also be a source of noise in RAM reactions. Those events are rare $[14,24]$ but can occur, resulting in ssDNA circles that are identical to circles formed after ligation on homologous template DNA. Likewise, RAM products generated from circles formed on nonhomologous targets, apart from their late Rts, are indistinguishable from homologous ligation signals and cannot be differentiated by product RAM product analysis from homologous target ligation signals. The assay as described seeks to minimize those reactions by optimizing the gene-specific ends of the C-probe, by the choice of target strand and C-probe (see below), and by limiting ligation reaction time and conditions.

Mismatch base pairings, and the context in which they exist, exhibit a range of thermodynamic favorabilities [29], and SNP discrimination should be greatest where the mismatch is least stable. For both G>A mutations (FV and PT), the plus strand (Wt SNP base $=\mathrm{G}$ ) was chosen as the $\mathrm{Wt}$ target, that is, the $\mathrm{WtC}+, \mathrm{MtC}-$ format, where the $\mathrm{Wt} \mathrm{C}$ probes are directed towards the Wt allele on the plus strand (Figure 2(a)). With this arrangement a mismatched C-probe and SNP base result in a C:A pairing. The alternative strategy, $\mathrm{MtC}+$, WtC-, where the Mt C-probe hybridizes to the $\mathrm{Mt}$ allele plus strand, would result in a more stable G:T $[29,30]$ mismatch. In the case of the MTHFR SNPs, the MTHFR 677 C-T and the MTHFR $1298 \mathrm{~A}-\mathrm{C}(\mathrm{C}>\mathrm{T}$ and $\mathrm{A}>\mathrm{C}$, resp.), the $\mathrm{MtC}+, \mathrm{WtC}-$ design was employed to create C:A and A:G mismatches as opposed to G:T and C:T, respectively. In the $\mathrm{MtC}+$, WtC- format the MTHFR $677 \mathrm{C}-\mathrm{T}$ base mismatch 
(C:A) is the same as would occur in PT 20210 G-A and FV 1691 G-A C-probe: target disparities. The format $\mathrm{MtC}+, \mathrm{WtC}-$, chosen for MTHFR 1298 A-C, resulted in a G:A mismatch. This difference was thermodynamically similar to the C:T mismatch that would be generated by the alternative $\mathrm{WtC}+$, $\mathrm{MtC}$ - format [29]. We expect that minimizing ligation on a nonhomologous target should make ssDNA circle formation a rare event and should produce a response time that is much later than Rts from the equivalent homologous ligation. We did not compare both $\mathrm{WtC}+, \mathrm{MtC}-$ and $\mathrm{MtC}+\mathrm{WtC}-$ probe: target formats for each SNP to test the prediction for base mismatch stability.

The lack of noise in nonhomologous assays makes postreaction signal analysis relatively straightforward; simple twodimensional plots of Mt versus Wt response times allow ready visualization of homozygotes or heterozygotes (Figures 3 and 4).

Padlock probes, in the molecular inversion probe form, have been applied for SNP detection, initially by Hardenbol et al. [15] and are now widely applied. In these assays, thousands of SNPs are assayed from a single sample with an array readout. In the clinical lab, by contrast, the number of samples is usually much greater than the number of targets to be assayed, making the array approach uneconomical. The process described here is appropriate for a clinical laboratory with sufficient sample processing demands to warrant moderate scale automation. To our knowledge, the only comparable process description, where C-probes are annealed directly to genomic DNA and assayed by isothermal RAM reactions, is Faruqi et al. [14] although no automation solutions were presented there and other assay differences exist.

Each the C-probe ligation and RAM assay presented here may provide distinct advantages over other nucleic acid detection assay formats. C-probe annealing makes the recognition events that flank the SNP nonindependent, in contrast to annealing unlinked sequences as in PCR. Edwards et al. [31] demonstrated SNP determination in a hexaploid wheat where PCR SNP determination was not feasible. Although a long C-probe sequence is required, the probe concentration in each hybridization is low as once the $5^{\prime}$ terminus of the $\mathrm{C}$-probe binds to the target, the local concentration of the $3^{\prime}$ terminus, in the proximity of the target, increases substantially. Magnetic bead processing on an automated platform makes the process as described here well-suited for moderate to high sample numbers, and further automation is possible. The combination of an automated platform with capture-probes that bind coated magnetic beads makes the assay workflow efficient, and the RAM reaction that detects ligated C-probe circles is low-noise, isothermal, and sensitive.

\section{Conclusion}

We have further evaluated a mostly automated, bead-based, isothermal RAM SNP assay by testing a panel of clinically relevant SNP targets in real clinical samples. In a blind study, we performed 280 SNP assays on 44 clinical samples and the results were in complete agreement with results from commercial FDA approved kits. These results suggest that this approach can meet a need in the clinical lab where moderate numbers of samples can be tested against specific clinically relevant panels of SNP targets.

\section{Conflict of Interests}

The authors declare that there is no conflict of interests regarding the publication of this paper.

\section{Acknowledgments}

The authors thank Mr. Raymond Nietupski for technical assistance, Ms. Cindy Rodzen for help with figures, and Dr. Harry McCoy for a critical reading of the paper. With our colleagues Dr. McCoy and Mr. Nietupski, the authors acknowledge with gratitude the contributions to this project of the late Dr. David J. Lane.

\section{References}

[1] J. H. Smith and T. P. Beals, "Detection of nucleic acid targets using ramified rolling circle DNA amplification: a single nucleotide polymorphism assay model," PLOS ONE, vol. 8, no. 5, Article ID e65053, 2013.

[2] F. R. Rosendaal, T. Koster, J. P. Vandenbroucke, and P. H. Reitsma, "High risk of thrombosis in patients homozygous for factor V Leiden (activated protein C resistance)," Blood, vol. 85, no. 6, pp. 1504-1508, 1995.

[3] S. R. Poort, F. R. Rosendaal, P. H. Reitsma, and R. M. Bertina, "A common genetic variation in the $3^{\prime}$-untranslated region of the prothrombin gene is associated with elevated plasma prothrombin levels and an increase in venous thrombosis," Blood, vol. 88, no. 10, pp. 3698-3703, 1996.

[4] P. Frosst, H. J. Blom, R. Milos et al., "A candidate genetic risk factor for vascular disease: a common mutation in methylenetetrahydrofolate reductase," Nature Genetics, vol. 10, no. 1, pp. 111113, 1995.

[5] I. Weisberg, P. Tran, B. Christensen, S. Sibani, and R. Rozen, "A second genetic polymorphism in methylenetetrahydrofolate reductase (MTHFR) associated with decreased enzyme activity," Molecular Genetics and Metabolism, vol. 64, no. 3, pp. 169172, 1998.

[6] N. Ilhan, M. Kucuksu, D. Kaman, N. Ilhan, and Y. Ozbay, "The $677 \mathrm{C} / \mathrm{T}$ MTHFR polymorphism is associated with essential hypertension, coronary artery disease, and higher homocysteine levels," Archives of Medical Research, vol. 39, no. 1, pp. 125130, 2008.

[7] S. Khan and J. D. Dickerman, "Hereditary thrombophilia," Thrombosis Journal, vol. 4, article 15, 2006.

[8] X. Yang, "Use of functional genomics to identify candidate genes underlying human genetic association studies of vascular diseases," Arteriosclerosis, Thrombosis, and Vascular Biology, vol. 32, no. 2, pp. 216-222, 2012.

[9] B. Furie and B. C. Furie, "Mechanisms of thrombus formation," New England Journal of Medicine, vol. 359, no. 9, pp. 938-949, 2008.

[10] N. Mtiraoui, W. Zammiti, L. Ghazouani et al., "Methylenetetrahydrofolate reductase C677T and A1298C polymorphism and changes in homocysteine concentrations in women with 
idiopathic recurrent pregnancy losses," Reproduction, vol. 131, no. 2, pp. 395-401, 2006.

[11] J. Tavakkoly Bazzaz, M. Shojapoor, H. Nazem et al., "Methylenetetrahydrofolate reductase gene polymorphism in diabetes and obesity," Molecular Biology Reports, vol. 37, no. 1, pp. 105-109, 2010.

[12] G. Chauhan, I. Kaur, R. Tabassum et al., "Common variants of homocysteine metabolism pathway genes and risk of type 2 diabetes and related traits in Indians," Experimental Diabetes Research, vol. 2012, Article ID 960318, 25 pages, 2012.

[13] T. P. Beals, J. H. Smith, R. M. Nietupski, and D. J. Lane, "A mechanism for ramified rolling circle amplification," $B M C$ Molecular Biology, vol. 11, article 94, 2010.

[14] F. A. Faruqi, S. Hosono, M. D. Driscoll et al., "High-throughput genotyping of single nucleotide polymorphisms with rolling circle amplification," BMC Genomics, vol. 2, article 4, 2001.

[15] P. Hardenbol, J. Banér, M. Jain et al., "Multiplexed genotyping with sequence-tagged molecular inversion probes," Nature Biotechnology, vol. 21, no. 6, pp. 673-678, 2003.

[16] M. Nilsson, H. Malmgren, M. Samiotaki, M. Kwiatkowski, B. P. Chowdhary, and U. Landegren, "Padlock probes: circularizing oligonucleotides for localized DNA detection," Science, vol. 265, no. 5181, pp. 2085-2088, 1994.

[17] D. Yong Zhang, M. Brandwein, T. Chun Hung Hsuih, and H. $\mathrm{Li}$, "Amplification of target-specific, ligation-dependent circular probe," Gene, vol. 211, no. 2, pp. 277-285, 1998.

[18] P. M. Lizardi, X. Huang, Z. Zhu, P. Bray-Ward, D. C. Thomas, and D. C. Ward, "Mutation detection and single-molecule counting using isothermal rolling-circle amplification," Nature Genetics, vol. 19, no. 3, pp. 225-232, 1998.

[19] S. Rozen and H. Skaletsky, "Primer3 on the WWW for general users and for biologist programmers," in Bioinformatics Methods and Protocols, S. Misener and S. A. Krawetz, Eds., pp. 365386, Humana Press, Totowa, NJ, USA, 2000.

[20] Roche, "MagnaPure LC DNA isolation kit," 2010, https:// cssportal.roche.com/LFR_PublicDocs/ras/03003990001_en_18 .pdf.

[21] R. C. Team, "R: a language and environment for statistical computing," R Foundation for Statistical Computing, Vienna, Austria, 2012, http://www.r-project.org/ .

[22] L. Komsta, "Outliers," 2011.

[23] J. L. Burg, P. B. Cahill, M. Kutter, J. E. Stefano, and D. E. Mahan, "Real-time fluorescence detection of RNA amplified by $\mathrm{Q} \beta$ replicase," Analytical Biochemistry, vol. 230, no. 2, pp. 263-272, 1995.

[24] M. Nilsson, J. Banér, M. Mendel-Hartvig et al., "Making ends meet in genetic analysis using padlock probes," Human Mutation, vol. 19, no. 4, pp. 410-415, 2002.

[25] T. P. Beals, Application to RAM Amplification of Real-time Analysis Methodologies Developed for PCR, qPCR BioStatistics \& BioInformatics, Freising, Germany, 2009.

[26] W. Abu Al-Soud and P. Rådström, "Effects of amplification facilitators on diagnostic PCR in the presence of blood, feces, and meat," Journal of Clinical Microbiology, vol. 38, no. 12, pp. 4463-4470, 2000.

[27] T. Demeke and G. R. Jenkins, "Influence of DNA extraction methods, PCR inhibitors and quantification methods on realtime PCR assay of biotechnology-derived traits," Analytical and Bioanalytical Chemistry, vol. 396, no. 6, pp. 1977-1990, 2010.

[28] A. D. Hale, J. Green, and D. W. G. Brown, "Comparison of four RNA extraction methods for the detection of small round structured viruses in faecal specimens," Journal of Virological Methods, vol. 57, no. 2, pp. 195-201, 1996.

[29] T. Naiser, J. Kayser, T. Mai, W. Michel, and A. Ott, "Position dependent mismatch discrimination on DNA microarraysexperiments and model," BMC Bioinformatics, vol. 9, article 509, 2008.

[30] H. T. Allawi and J. SantaLucia Jr., "NMR solution structure of a DNA dodecamer containing single G·T mismatches," Nucleic Acids Research, vol. 26, no. 21, pp. 4925-4934, 1998.

[31] K. J. Edwards, A. L. Reid, J. A. Coghill, S. T. Berry, and G. L. A. Barker, "Multiplex single nucleotide polymorphism (SNP)based genotyping in allohexaploid wheat using padlock probes," Plant Biotechnology Journal, vol. 7, no. 4, pp. 375-390, 2009. 

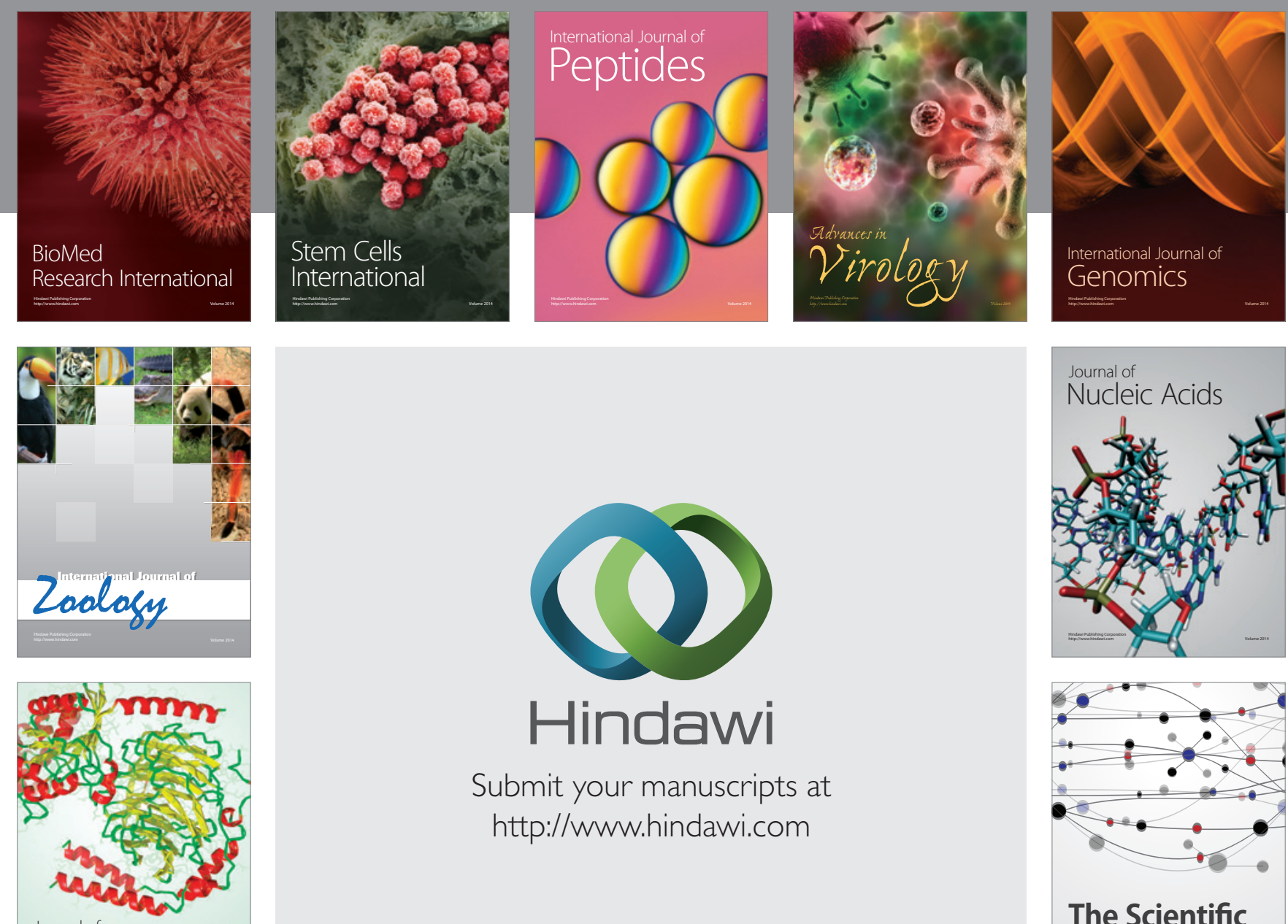

Submit your manuscripts at

http://www.hindawi.com

Journal of
Signal Transduction
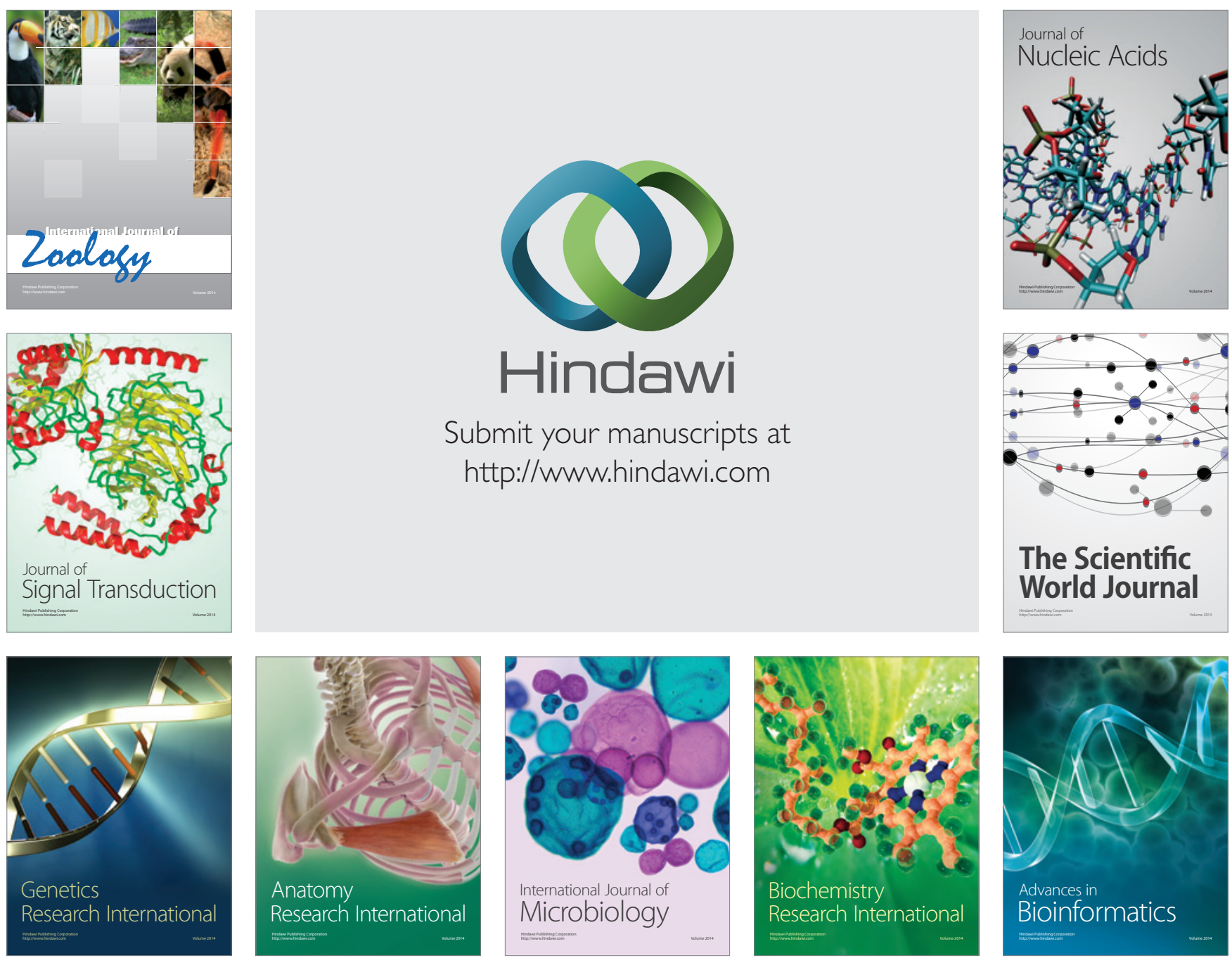

The Scientific World Journal
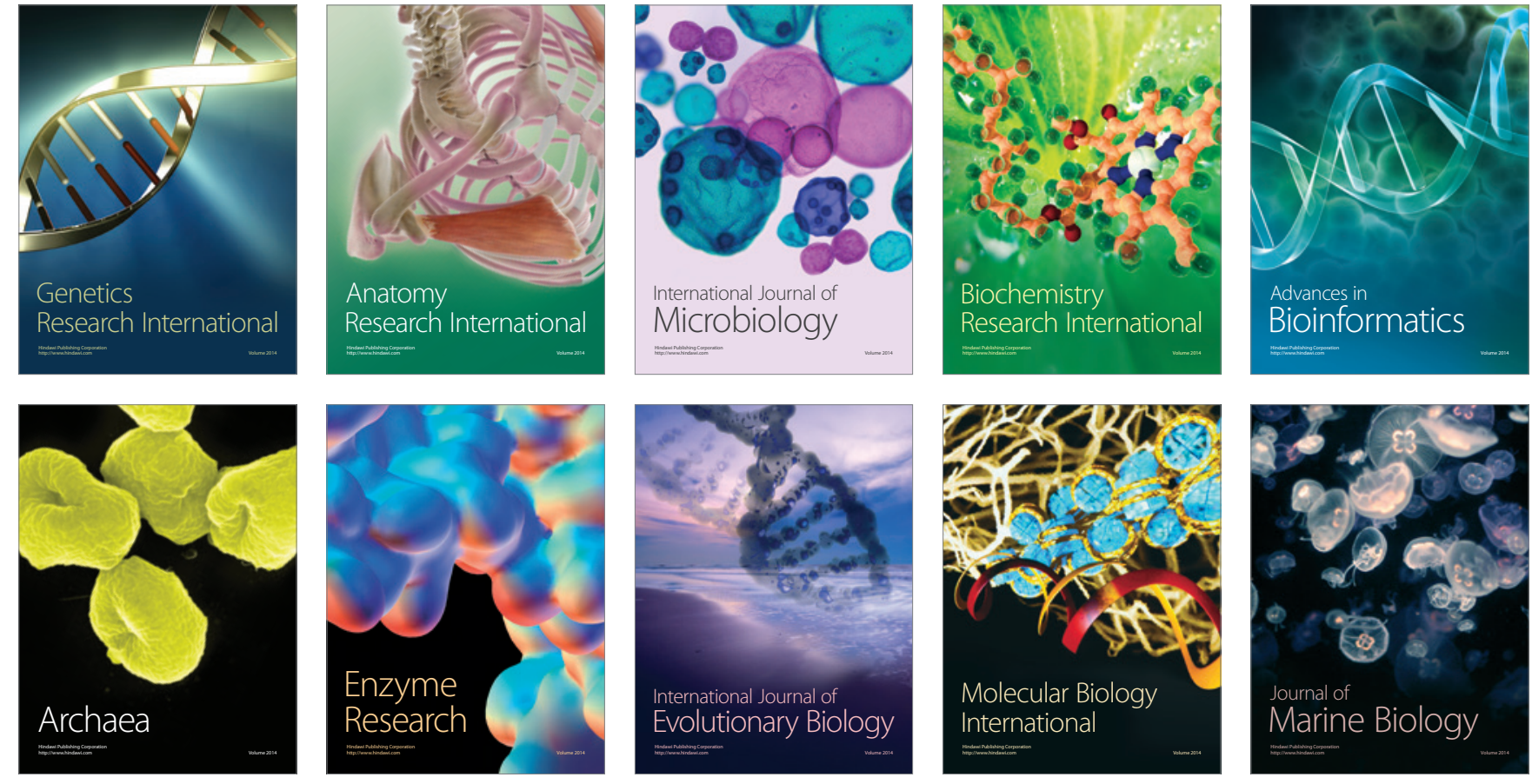